\title{
Laparoscopic splenectomy for Immune thrombocytopenic purpura (ITP)
}

\author{
Ahmed H. Ali, FRCS; Ahmed Nafei, MD; Tarek Youssef Ahmed, MD, MRCS
}

Department of Surgery, Ain Shams University, Cairo, Egypt.

Background: In recent years laparoscopic splenectomy (LS) has gained acceptance as a viable alternative to open splenectomy. Laparoscopic splenectomy is evolving and may become the standard operative method for the treatment of the problem spleen.

Patients and methods: From July 2012 till December 2013, 20 patients with clinical immune thrombocytopenic purpura (ITP) were referred as surgical candidates. Two of them (10\%) were converted to open, and the other 18 underwent successful LS. The operative approach was performed with the patient in the right lateral decubitus position, with extensive use of the ultrasonic dissecting shears.

Results: There were 20 patients in the study, twelve women and eight men with a mean age of 28 years (18-38). Immune thrombocytopenic purpura (ITP) was the indication for surgery. Laparoscopic splenectomy was intended in all patients and successfully completed in 18 patients (90\%). Two cases (10\%) required conversion to open splenectomy, due to intraoperative hemorrhage which could not be controlled laparoscopically. Length of hospital stay (LOS) was $2.3 \pm 0.8$ days. The two major complications (10\%) were intraoperative hemorrhage which required conversion to open splenectomy. The two minor complications were trocar site infection and hernia. The average operative times (150 $\pm 48 \mathrm{~min})$ improved as the study progressed. This improvement was believed to be due to the learning curve.

Conclusion: Laparoscopic splenectomy (LS) may be considered the standard of care for patients who require splenectomy for ITP. Laparoscopic splenectomy results in less patient disability, shorter hospitalization, and probably less perioperative morbidity

Key words: Spleen, splenectomy, laparoscopy, ITP, thrombocytopenic purpura.

\section{Introduction:}

Laparoscopic splenectomy was first successfully performed and published by Delaitre and Maignien in 1991. ${ }^{1}$ Since then, laparoscopic splenectomy has become acceptedassafe and effective forbenignsplenic disorders such as immune thrombocytopenic purpura (ITP). ${ }^{2}$ Laparoscopic splenectomy is a safe procedure and can provide less postoperative morbidity in experienced hand as open splenectomy, and most cases that require splenectomy can be treated laparosopically. Laparoscopic splenectomy is a useful method for reducing hospital stay, complications and early return to normal activity. With better training in minimal access surgery now available, the time has arrived for it to take its place in modern surgery. ${ }^{3}$ Several studies from centers dedicated to laparoscopy have shown the well-known advantages of minimally invasive surgery, e.g., decreased postoperative pain, shorter hospital stay, and reduced recovery time. ${ }^{4}$ A recently published meta-analysis revealed a significantly lower complication rate after laparoscopic versus open splenectomy in particular; pulmonary complications, wound infections, and systemic infectious complications were decreased. ${ }^{5}$ Laparoscopic splenectomy (LS) rapidly gained widespread acceptance for the treatment of nontraumatic diseases of the spleen during the last 10 years. Increased technical skills and technical developments have extended the indications 
for the laparoscopic removal of the spleen. ${ }^{6}$ Hematologic diseases such as idiopathic thrombocytopenic purpura (ITP) and thrombotic thrombocytopenic purpura (TTP) with normal or only moderately enlarged spleens are still the most common indications for a laparoscopic splenectomy. ${ }^{7}$ Massively enlarged spleens are often difficult to handle intraoperatively, and there is concern about higher conversion rates and higher morbidity also retrieving a large specimen may require a long incision so that the benefits of minimally invasive access of LS are wasted. ${ }^{8}$ However, the spleen can be morcellated and removed in a bag, with minimal risk of spleen cell spreading. Hence, patients with a bulky spleen revealing a long axis of more than $20 \mathrm{~cm}$ are commonly excluded from a laparoscopic approach. ${ }^{9}$ Other authors advocate open splenectomy (OS) in patients with a spleen larger than $1 \mathrm{~kg}$ or a splenic axis greater than $15 \mathrm{~cm}^{10}$

\section{Patients and methods:}

Patients: From July 2012 till December 2013, 20 patients with clinical ITP underwent laparoscopic splenectomy (LS) at Ain Shams University hospitals. The decision to perform splenectomy was made by both the surgeon and hematologist/oncologist. Standard preoperative laboratory studies and other tests were obtained, including a type and screen or cross match for blood products. Ultrasound examination was obtained preoperatively to assess spleen size (maximum pole length), measured as the joining line between the two organ poles and was divided into three categories: (a) normal spleen $(<11 \mathrm{~cm})$, (b) moderate splenomegaly $(11-20 \mathrm{~cm})$, or (c) severe splenomegaly $(>20 \mathrm{~cm})$. Laparoscopic splenectomy (LS) was performed for patients with category a and b while patients with severe splenomegaly were excluded from this study. A radiologist was also asked to try to identify accessory spleens in all cases. Absolute contraindications for laparoscopic splenectomy in this series included portal hypertension, severe cardiopulmonary disease, and uncorrectable coagulopathy. Operative time, platelet count, conversion rate and length of hospital stay were recorded

\section{Operative techniques:}

Preoperative preparation: All patients received polyvalent pneumococcal and Haemophilus influenza type $b$ vaccines two weeks before surgery. Perioperative antibiotic coverage in the form of a single dose of a third-generation cephalosporin was given routinely.

Surgical technique Figures (1-4): Informed consent for surgery was obtained with the understanding that conversion to open splenectomy may occur. After induction of general anesthesia and endotracheal intubation, a nasogastric or orogastric tube was placed, a urinary drainage catheter was inserted, and pneumatic compression stockings were applied. The operative approach for LS was performed with the patient in the right lateral decubitus position, with extensive use of the ultrasonic dissecting shears, late division of the splenic hilum, and extraction bag of the spleen at a subcostal trocar site. The left upper quadrant, splenocolic ligament, splenic hilum, and lesser sac were routinely explored for accessory spleens. Ultrasonic dissection of retrosplenic peritoneal attachments, splenophrenic ligament, and the short gastric vessels allowed anteromedial mobilization of the spleen. Once elevated, the splenic hilum was divided by linear cutting stapler, and the organ was placed in the extraction bag, with emphasis on minimal parenchymal handling. The specimen was mechanically fracturemorcellated via the exteriorized extraction bag. Care was taken not to spill any tissue fragments. After adequate hemostasis, the fascia and wounds were closed. The lateral position was clearly the best. And most studies support the use of lateral and semilateral position. The spleen was located deep in the left hypochondrium and a key maneuver for splenectomy was the mobilization of the organ. Lateral and semilateral position took the advantage of gravity and avoided spleen grasping and stretching. The full lateral approach with adequate fixation of the patient to the table allowed exaggerating of 
the lateral tilt of the table and better access to the posterior or anterior face of the spleen. A minor drawback of this position was in case of conversion to open and the need to change the position to supine but the advantages were outweighing these disadvantages.

Adequate pain relief was successfully achieved with local anesthetics injected around trocar sites, and with oral and intravenous analgesic agents. All patients tolerated clear oral liquid on the evening of surgery. The majority of the patients returned to unrestricted activity within a week.

\section{Statistics:}

All data collected were analyzed using SPSS 11. for Windows statistical software.

\section{Results:}

There were 20 patients in the study, twelve women and eight men with a mean age of 28 years (18-38). Immune thrombocytopenic purpura (ITP) was the indication for surgery Table (1).

The presence of purpura and/or petechial rash was the most common presentation, and recurrent episodes of ITP were the primary indication for surgery Table (2).

The majorities of patients responded to preoperative treatment and were able to attain platelet counts $>25,000 / \mathrm{mm}^{3}$ at the time of surgery. LS were uniformly successful in inducing clinical remission in this group. In two patients with counts that remained $<25,000 / \mathrm{mm}^{3}$ in spite of aggressive medical treatment, the effectiveness of LS fell to $50 \%$ Table (3).

Laparoscopic splenectomy was intended in all patients and successfully completed in 18 patients (90\%). Two cases (10\%) required conversion to open splenectomy, due to intraoperative hemorrhage which could not be controlled laparoscopically. Length of hospital stay (LOS) was $2.3 \pm 0.8$ days. There were no deaths. The two major complications (10\%) were intraoperative hemorrhage which required conversion to open splenectomy. The two minor complications were trocar site infection and hernia. The average operative times (150 $\pm 48 \mathrm{~min})$ and estimated blood loss (175 \pm 135$)$ were improved as the study progressed. This improvement was believed to be due to the learning curve and to the use of the ultrasonic dissecting shears Table (4).

\section{Discussion:}

By Deltaire laparoscopic splenectomy was first reported at the end of 1991. There are now quite a number of large series from around the world which confirm that the laparoscopic approach to splenectomy is the treatment of choice for the majority of patients. ${ }^{11}$ Laparoscopic splenectomy has become a definitive alternative to open surgery for removal of the non enlarged spleen and mild to moderate enlarged spleen. This particularly in ITP, the most frequent indication for splenectomy in a general hospital. ${ }^{12}$ In this study ITP was the only indication for laparoscopic splenectomy. The precise size limit for attempting laparoscopic splenectomy is still under evaluation, but it would appear that spleens 28 to $30 \mathrm{~cm}$ or greater in longitudinal dimension and 3000 gm or more in weight are best approached in open fashion because of the low success rate with the laparoscopic approach. ${ }^{13}$ This is comparable with this study as LS was done to spleen size less than $20 \mathrm{~cm}$. Another study showed that most patients with moderately enlarged spleens (15-20 cm in longitudinal dimension or $<1000 \mathrm{~g}$ in weight) can usually be resected laparoscopically. ${ }^{11}$ Considerable experience should be developed with laparoscopic splenectomy in non enlarged spleens before attempting this procedure in patients with marked splenomegaly. ${ }^{14}$ Splenectomy is indicated in adult patient who failed steroid therapy. In this study patients with preoperative platelet count $>25,000$ showed remission rate $(100 \%) 16 / 16$ while patients with preoperative platelet count $<25,000$ showed remission rate $(50 \%) 2 / 4$ which is comparable to ronald and Alfons study which showed permanent improvement of platelet count in about $80 \%$ of patients. 15

Lee and Kim, 1997 performed a total of 53 laparoscopic splenectomeis. Among them 40 patients had immune thrombocytopenic purpura 35 were females and 5 patients were 
males. The mean age was 34, varying from 17 to 56. The mean hospital stay was 5 days. There was no perioperative mortality; but in 2 cases they had postoperative subpherenic abscesses which were successfully drained by catheter drainage. Since undergoing laparoscopic splenectomy, 28 patients (70\%) were weaned effectively from their steroid medications. 8 patients (20\%) have been on small doses of steroid, and 4 patients (10\%) have been on the same doses of steroid with no response. The patient group with rapidly increasing platelet count after splenectomy showed a statistically significant relation with the complete response group. Their opinion were that laparoscopic splenectomy is a safe and reasonable operative procedure for patients with immune thrombocytopenic purpura. ${ }^{16}$ In this study the average age was 28 (range 18-38), length of hospital stay (LOS) was $2.3 \pm 0.8$ days. The two major complications (10\%) were intraoperative hemorrhage which required conversion to open splenectomy. The two minor complications were trocar site infection and hernia.

Lefor et al., 1993 reviewed their initial experience with laparoscopic splenectomy in patients with hematologic diseases. They found that laparoscopic splenectomy was attempted in 43 patients and successfully completed in 35 (81\%). Therapeutic platelet response to splenectomy occurred in $82 \%$ of patients with immune thrombocytopenic purpura undergoing successful laparoscopic splenectomy. The morbidity rate was $11.6 \%$ (5 of 43 patients). Mean length of stay was 2.7 days after laparoscopic splenectomy. Their opinion were that laparoscopic splenectomy may be performed with efficacy and morbidity rates comparable to those of open splenectomy for hematologic diseases, and it appears to retain other patient benefits of laparoscopic surgery. ${ }^{17}$

Katkhouda, et al., 1998 studied the safety and efficacy of laparoscopic splenectomy (LS) in patients with predominantly benign hematologic disorders in one hundred three consecutive patients who underwent LS. They found that indications were idiopathic thrombocytopenic purpura (ITP), hereditary spherocytosis, autoimmune hemolytic anemia, and thrombotic thrombocytopenic purpura. Mean spleen size was $14 \mathrm{~cm}$ and mean weight was $263 \mathrm{~g}$. There were no deaths. Complications occurred in six patients, one requiring a second procedure for small bowel obstruction. Six patients received transfusions, and four procedures were converted to open splenectomy for bleeding. Mean surgical time was 161 minutes and was greater in the first 10 cases than the last. ${ }^{10}$ Mean postsurgical stay was 2.5 days. Thrombocytopenia resolved after surgery in $84 \%$ of patients with ITP, and hematocrit levels increased significantly in $70 \%$ of patients with chronic hemolytic anemias. A positive response was noted in $92 \%$ of patients with hereditary spherocytosis, without relapse for the duration of the observation. ITP relapsed in four patients during follow-up, three within 12 months. Their opinions were that LS can be performed safely and effectively in a teaching institution. LS should become the technique of choice for treatment of intractable benign hematologic disease. ${ }^{18}$ In this study laparoscopic splenectomy (LS) was performed for patients with splenic size $<11 \mathrm{~cm}$. Laparoscopic splenectomy was successfully completed in 18 patients (90\%). Two cases (10\%) required conversion to open splenectomy, due to intraoperative hemorrhage which could not be controlled laparoscopically. Length of hospital stay (LOS) was $2.3 \pm 0.8$ days. A positive response was noted in $16 / 16$ patients with platelet counts $>25,000 / \mathrm{mm}^{3}$ at the time of surgery and LS was uniformly successful in inducing clinical remission. In two patients with counts that remained $<25,000 / \mathrm{mm}^{3}$ in spite of aggressive medical treatment, the effectiveness of LS fell to $50 \%$. There were no deaths. The two major complications (10\%) were intraoperative hemorrhage which required conversion to open splenectomy. The two minor complications were trocar site infection and hernia. The average operative times (150 $\pm 48 \mathrm{~min})$ were improved as the study progressed. This improvement was believed to be due to the learning curve and to the use of the ultrasonic dissecting shears. 


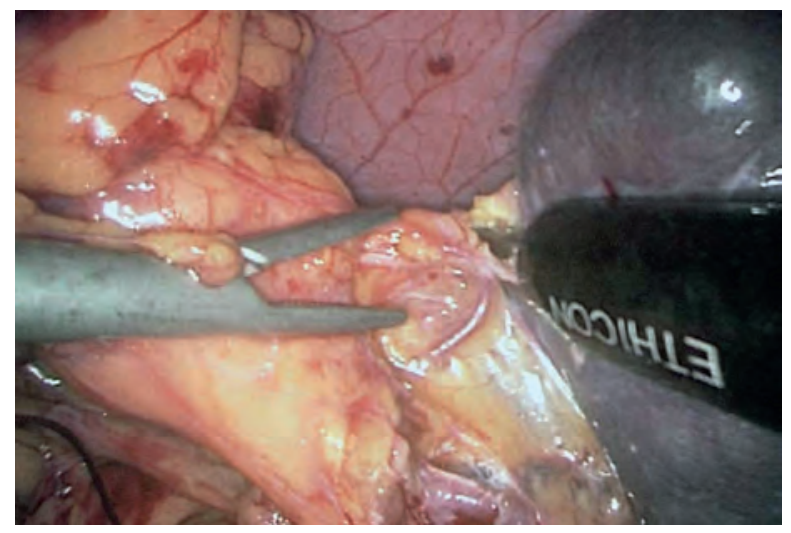

Figure (1): Division of the short gastric vessels with harmonic scalpel.

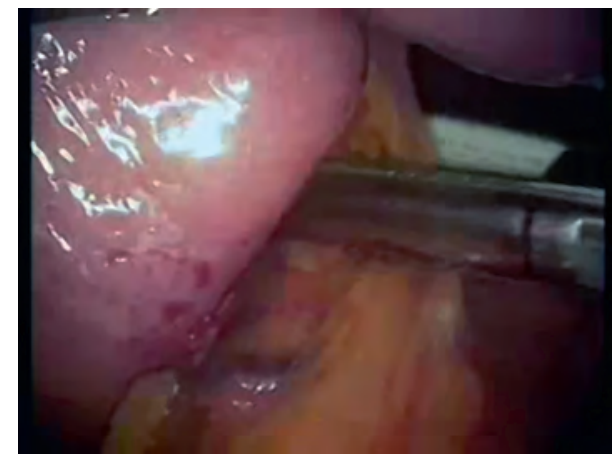

Figure (2): Division of splenic hilum by linear cutting stapler.

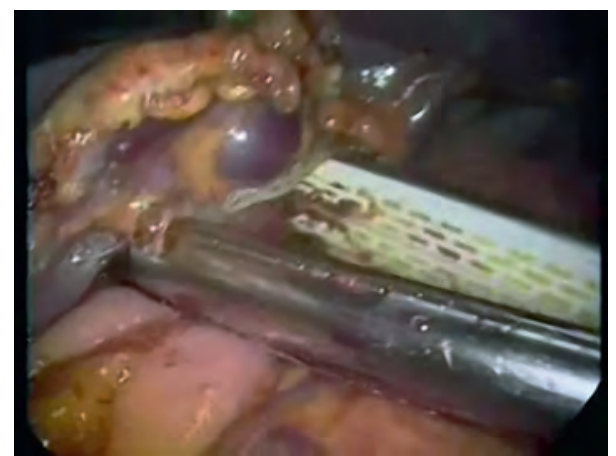

Figure (3): Division of splenic hilum by linear cutting stapler.

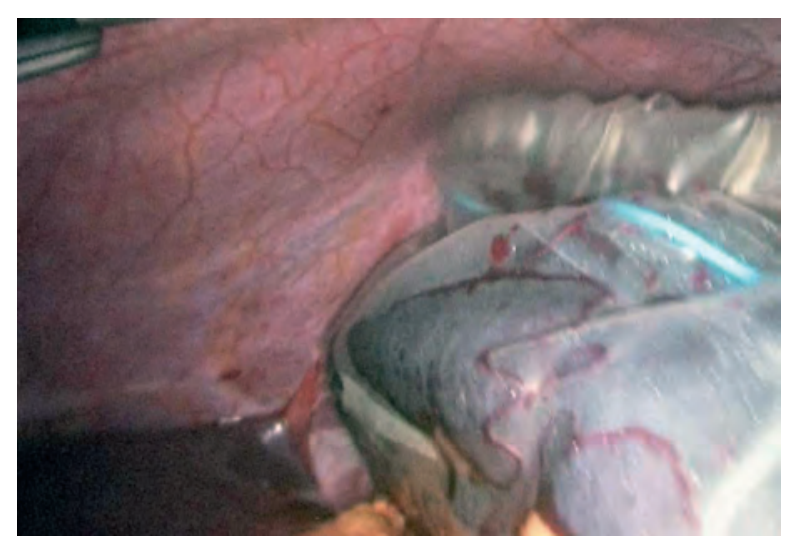

Figure (4): Spleen in retrieval bag.

Table 1: Patient demographics.

\begin{tabular}{|l|l|}
\hline No. of patients & 20 \\
\hline Age (yr) & 28 (range 18-38) \\
\hline Sex & \\
Male & $8(40 \%)$ \\
Female & $12(60 \%)$ \\
\hline
\end{tabular}

Misawa et al., 2009 studied and evaluated the outcome of patients undergoing laparoscopic splenectomy (LS) on 52 patients undergoing LS compared to 28 concurrently treated open splenectomy patients (OS). These patients did not differ with regard to age, gender, body, or splenic weights. The operative time was longer in the LS patients 
Table 2: Indications for surgery.

\begin{tabular}{|l|lc|}
\hline Indications for surgery & Recurrent episodes of ITP & 18 \\
& Steroid-intolerant & 1 \\
& Steroid-resistant ITP & 1 \\
\hline
\end{tabular}

Table 3: Surgical results related to platelet count.

\begin{tabular}{|c|l|}
\hline Preop platelet count & Remission rate \\
\hline$>25,000$ & $16 / 16(100 \%)$ \\
\hline$<25,000$ & $2 / 4(50 \%)$ \\
\hline
\end{tabular}

Table 4: Surgical results and complications.

\begin{tabular}{|l|l|}
\hline Length of stay (days) & $2.3 \pm 0.8$ (range, $1-5)$ \\
\hline Major complications & $2(10 \%)$ \\
\hline Minor complications & $2(10 \%)$ \\
\hline OR time (min) & $150 \pm 48$ (range, $90-240)$ \\
\hline Est. blood loss (ml) & $175 \pm 135$ (range, 50-500) \\
\hline
\end{tabular}

(mean 196 vs. $156 \mathrm{~min}$ ), but the length of stay were shorter in the LS group. Six patients required conversion to OS, four occurring in the first 11 patients treated (overall conversion rate of $11 \%)$. Three patients died from complications related to their underlying disease. ${ }^{19}$ In this study the average operative time was (150 $\pm 48 \mathrm{~min})$. Length of hospital stay (LOS) was $2.3 \pm 0.8$ days. There were no deaths. The two major complications (10\%) were intraoperative hemorrhage which required conversion to open splenectomy. The two minor complications were trocar site infection and hernia.

\section{Conclusion :}

Laparoscopic splenectomy could be considered the standard of care for patients who require splenectomy for ITP, with primary splenic manifestations. Laparoscopic splenectomy results in less patient disability, shorter hospitalization, and probably less perioperative morbidity.

\section{Reference:}

1- Delaitre B, Maignien B: Splenectomy by the coelioscopic approach: Report of a case. Presse Med 1991; 20: 2263.
2- Brodsky JA, Brody FJ, Walsh RM: Laparoscopic splenectomy. Surg Endosc 2002; 16: 851-854.

3- Knauer EM, Ailawadi G, Yahanda A: 101 laparoscopic splenectomies for the treatment of benign and malignant haematologic disorders. Am J Surg 2003; 52(5): 500-504.

4- Grahn SW, Alvarez J, Kirkwood K: Trends in laparoscopic splenectomy for massive splenomegaly. Arch Surg 2006; 141(8): 755-761.

5- Winslow ER, Brunt LM: Perioperative outcomes of laparoscopic versus open splenectomy: A meta-analysis with emphasis on complications. Surgery 2003; 134: 647-655.

6- Katz SC, Pachter HL: Indications for splenectomy. Am J Surg 2006; 72(7): 565-580.

7- Silecchia G, Raparelli L, Casella G: Laparoscopic splenectomy for non-traumatic diseases. Minerva Chir 2005; 60(5): 363-374.

8- Mahon D, Rhodes M: Laparoscopic splenectomy: Size matters. Ann R Coll Surg Eng 2003; 1 85(4): 248-251.

9- Uranues S, Alimoglu O: Laparoscopic surgery of the spleen. Surg Clin North Am 2005; 85: 75-90.

10- Boddy AP, Mahon D, Rhodes M: Does open surgery continues to have a role in elective 
splenectomy? Surg Endosc 2006; 20: 1094-1098.

11- Park AE, Birgisson G, Mastrangelo MJ: Laparoscopic splenectomy, outcomes and lessons learned from over 200 cases. Surgery (St. Louis) 2000; 128: 660-667.

12- Whitman ED, Brunt LM, Bruce V, Maruice E, Douglas O: Laparoscopic surgery of abdomen. New York. 2004; PP: 238-254.

13- Schlachta CM, Poulin EC, Mamazza J: Laparoscopic splenectomy for hematologic malignancies. Ann Surg 1998; 228: 35-39.

14- Targarona E, Trias M: Laparoscopic treatment of splenic injuries. Semin Laparosc Surg 1996; 3: 44-49.

15- Ronald M, Ahmad A, Alfons P: Choice of laparoscopic techniques: In: controversies in laparoscopic surgery. Ahmad A, Michael G and Moshe Schein (Eds). New York. 2005;
PP: 305-307.

16- Lee WJ, Kim BR: Laparoscopic splenectomy for idiopathic thrombocytopenic purpura. Surg Endosc 1997; 7: 209-212.

17- Lefor AT, Melvin WS, Bailey RW: Laparoscopic splenectomy in the management of immune thrombocytopenia purpura. Surgery. 1993; 114(3): 613-618.

18- Katkhouda N, Hurwitz MB, Rivera RT: Laparoscopic splenectomy: Outcome and efficacy in 103 consecutive patients. Ann Surg 1998; 228: 568-578.

19- Misawa K, Takeyuki J: Minimizing intraoperative bleeding using a vessel-sealing system and splenic hilum hanging maneuver in laparoscopic splenectomy. Journal of Hepato-Biliary-Pancreatic Surgery 2009; 133: 16-20. 